\title{
Enhancement of pneumatic nebulization efficiency through application of an electric field
}

\author{
R. N. Savage and G. M. Hieftje $e^{\text {a) }}$ \\ Department of Chemistry, Indiana University, Bloomington, Indiana 47401 \\ (Received 18 May 1978; in final form, 5 July 1978)
}

A technique is described for reducing the size of aerosol droplets produced by a pneumatic nebulizer of the kind commonly used in flame or plasma spectrometry. The technique involves the application of an electric field to the tip of the nebulizer, resulting in an induced surface charge on the liquid being nebulized. In turn, the surface charge reduces the liquid's surface tension and results in the generation of a finer aerosol. In this study, the effect of the electric field is quantified in terms of the spatial dispersion and size distribution of aerosol droplets formed in the presence and absence of the field. Droplet size distributions, obtained using the $\mathrm{MgO}$ impression technique and analyzed using log-normal and upper-limit functions, reveal a $63 \%$ decrease in the volume mean droplet diameter in the field's presence. As a result, this system is expected to be useful in both flame and plasma spectrometry, where reduced droplet size can yield improved precision and freedom from interferences.

\section{INTRODUCTION}

One of the most important aspects of flame or plasma atomic spectrometry is the means and efficiency with which the sample solution is converted into an aerosol and carried to the analytical flame or plasma. In particular, droplet size plays a key role in determining the sensitivity, precision, and relative freedom from interferences that can be obtained with a flame or plasma. Small droplets will be quickly desolvated and will produce proportionally smaller solute particles which in turn will experience rapid and efficient vaporization. The mechanisms of desolvation and vaporization have been examined in some earlier studies ${ }^{1,2}$ which describe the complex sequence of events occurring in flames whereby sample solutions are transformed into free atoms. From these considerations, any modification which would enable a nebulizer to produce smaller droplets might aid in improving sensitivity and precision of flame or plasma spectrometry.

Several devices have been developed for converting a sample solution to an aerosol and range from simple right-angle pneumatic nebulizers ${ }^{3}$ to more complex devices such as ultrasonic nebulizers ${ }^{4}$ and electrostatic sprayers. ${ }^{5}$ Mavrodineanu ${ }^{6}$ has described these and other methods of sample introduction which are commonly employed in flame spectrometry. Of the many alternative techniques, pneumatic nebulization has become the most widely accepted means of sample introduction, because of its relatively low cost, high efficiency, and simplicity of operation. However, pneumatic nebulization produces relatively large droplets which vary widely in size.

In the present paper, a novel technique is described in which the size of pneumatically produced droplets is reduced by application of an intense electric field to the nebulized liquid. This new approach is simple to implement with nebulizers commonly employed in plasma or flame spectrometry and should find application in many laboratories. The performance of this new electric-field pneumatic nebulizer (EFPN) system is based upon the interaction of electric field forces and molecular forces within a charged liquid jet as it is pneumatically disrupted, resulting in an effective decrease in the surface tension of the liquid. This electric-field-induced reduction in surface tension leads to the pneumatic formation of smaller droplets by an established mechanism ${ }^{7,8}$ which describes the role that surface tension plays in pneumatic nebulization.

In the present investigation, aerosols were produced by an EFPN system constructed from a Beckman totalconsumption integral aspirator-burner. Because operation of the new nebulizer was the subject of study, no flame was employed; instead, the aerosol was measured directly and ambiguity thereby avoided. Photographic observation of the aerosols produced by the EFPN system reveals distinct perturbations in the spatial distribution of the droplets formed under the field's influence. These perturbations demonstrate that the droplets produced by this nebulizer are highly charged. In addition, droplet size distributions produced by the EFPN system were directly measured and compared, both with and without the electric field applied, and a $63 \%$ reduction in aerosol volume mean diameter was noted. As a necessary background for these studies, let us consider the basic mechanism of pneumatic nebulization and the influence that an electric field will exert on the droplet formation process.

\section{MECHANISM OF ELECTRIC-FIELD-ENHANCED NEBULIZATION}

The mechanism of pneumatic nebulization has been investigated and formulated by a number of authors ${ }^{9-12}$ 
and a series of theoretical treatments have described axisymmetric instabilities of flowing jets and their disintegration into droplets corresponding to the wavelength of fastest growth. However, our discussion can be restricted to those parameters involved in pneumatic nebulization which are affected by an electric field.

Nukiyama and Tanasawa ${ }^{8}$ correlated the size of droplets produced by concentric pneumatic nebulizers with the properties of the liquid being nebulized and with the relative velocities of the gases and liquids involved. Their results can be expressed as follows:

$$
D=\frac{585 \sqrt{S}}{v \sqrt{d}}+597\left(\frac{f}{\sqrt{d S}}\right)^{0.45}\left(1000 \frac{Q_{l}}{Q_{a}}\right)^{1.5}
$$

In Eq. (1), $D$ is the diameter $(\mu \mathrm{m})$ of a single droplet having the same volume-to-surface ratio as the entire population of droplets (termed the volume mean diameter), $S$ is the surface tension $(\mathrm{dyn} / \mathrm{cm}), d$ the density $\left(\mathrm{g} / \mathrm{cm}^{3}\right), f$ the viscosity $\left(\mathrm{dyn} / \mathrm{cm}^{2}\right), Q_{1}$ the flowrate $\left(\mathrm{cm}^{3} / \mathrm{s}\right)$ of the nebulized liquid, $v$ is the velocity $(\mathrm{m} / \mathrm{s})$, and $Q_{a}$ the flowrate $\left(\mathrm{cm}^{3} / \mathrm{s}\right)$ of the nebulizing gas. With most conventional pneumatic nebulizers, $Q_{1} / Q_{a}$ is insignificantly small; consequently, the first term in Eq. (1), involving the surface tension parameter, plays the dominant role in determining droplet sizes. Accordingly, any reduction of a solution's surface tension will produce a marked decrease in the size of the resulting droplets. This behavior is illustrated by the fact that an increase in the number of small droplets occurs in an aerosol when an organic solvent instead of an aqueous solvent is nebulized.

\section{Nature of electric field enhancement}

Schmid, Hurd, and Snavely ${ }^{13}$ reported that application of a strong electric field to a solution will reduce the solution's surface tension. This effect is well-recognized in the field of polarography, where application of a potential to a dropping mercury electrode alters the mercury-solution interfacial tension and consequently changes the mercury flow rate and drop time. Correspondingly, the application of an electric field should also reduce the size of pneumatically produced droplets. Mechanistically, the electric field forces oppose molecular forces within the droplet as it is formed, which results in a lowering of the droplet's surface tension and creates instabilities within the droplet. This complex interaction then leads to the breakup of the larger unstable droplets into smaller more stable droplets. In the case of aqueous solutions, surface tension reduction can be visualized as the result of an increase in the orientation of the water dipoles in the presence of an electric field. Similarly oriented dipoles will repel each other, thereby leading to a decrease in surface tension.

Instabilities created within a charged droplet can be linked to its acquisition of excess surface free energy when the droplet becomes charged; in turn, this free energy can be minimized by decreasing the droplet's charge-to-surface area ratio. A decrease in this ratio is conveniently implemented by fragmentation of a large droplet into a number of smaller droplets. Hence, the combination of electric field effects with pneumatic nebulization results in a system capable of substantially reducing the size of the droplets produced from a conventional pneumatic nebulizer.

Another technique closely related to the EFPN phenomenon just described deals with the breakup of charged liquid jets and is commonly called electrostatic spraying. Even though pneumatic forces do not play a role in electrostatic spraying, the mechanism of the influence of the electric field on pneumatic spraying is similar to that involved in electrostatic spraying. Consequently, the effect that an electric field will have on these processes should be similar.

The mechanism of electrostatic spraying has been studied extensively ${ }^{14-18}$ and a number of experimental and theoretical papers have appeared on the many processes involved in the breakup of charged liquid jets. ${ }^{19-25}$ Most of these investigations treat the observed surface instabilities as an opposition of molecular forces (e.g., surface tension) and electrical forces. In order to minimize surface energy, the molecular forces tend to decrease the surface-to-volume ratio (i.e., contract the liquid into a single sphere) whereas the energy associated with the electric field is minimized by increasing this ratio (i.e., shattering the liquid into tiny droplets). Consequently, as the electrical forces become dominant, a decrease occurs in the mean droplet diameter produced upon breakup of a liquid jet. This behavior has been confirmed by Huebner and others ${ }^{18,20,23}$ who found that the mean size of the droplets produced from such jets decreases with increasing applied potential. Disintegration of liquid jets at potentials of up to $25 \mathrm{kV}$ has been examined, but the electric fields created were actually weak due to large distances between electrodes. Distributions tabulated for mean droplet sizes in these studies range from 100 to $1000 \mu \mathrm{m}$, and size decreases on the order of $25 \%$ to $50 \%$, depending on the magnitude of the potential being applied, have been observed. Comparable reductions in droplet sizes might be expected for the EFPN system operated under similar electric field strength conditions; in fact, the EFPN system described herein yields a $63 \%$ decrease in the volume mean droplet diameter when a potential of $5.5 \mathrm{kV}$ is applied.

\section{EXPERIMENTAL}

A diagram of the EFPN system employed in the present work is shown in Fig. 1. The system is comprised of a charging electrode and a Beckman burner attached to a spatial translator and mounted within an insulated enclosure. To charge the droplets being formed, terminal B (connected to the nebulizer) is grounded, so the bulk analytical solution is not charged; however, a potential of $5.5 \mathrm{kV}$ is applied to terminal A by a high-voltage power supply (cf. Table I). Consequently, only that portion of the solution between the electrode and nebulizer is 


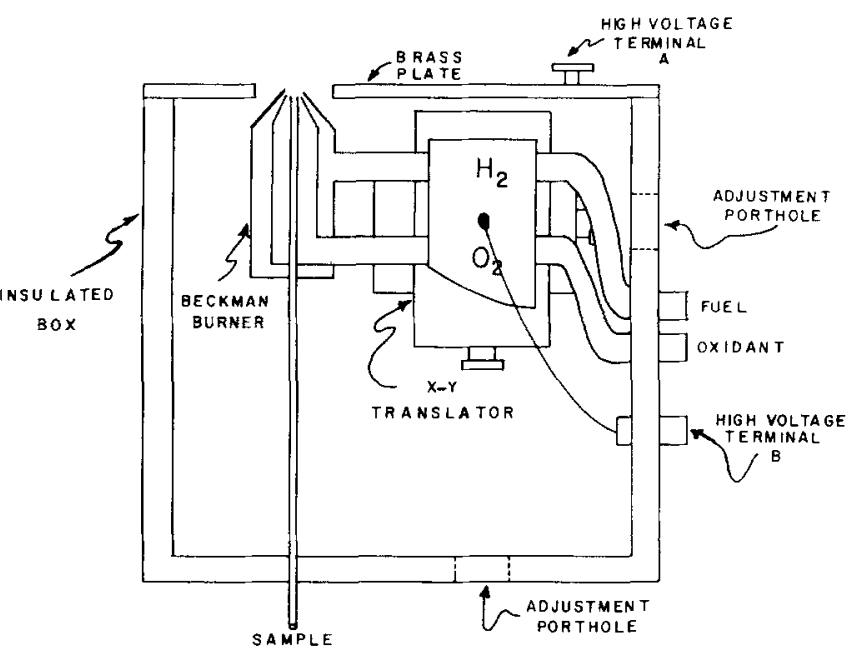

FIG. 1. Diagram of electric-field pneumatic nebulizer.

charged, thereby maximizing the electric field's effect. Care must be taken in the EFPN system design to minimize the distance between the nebulizer and terminal A, so the greatest electric field strength is generated. This goal was accomplished in the present study by positioning the nebulizer as close as possible to terminal A (approximately $1 \mathrm{~cm}$ ), without causing electrical breakdown.

With this arrangement, an electric field on the order of $5.5 \mathrm{kV} / \mathrm{cm}$ is created between the tip of the nebulizer and the charging electrode. We did not attempt to accurately measure or calculate the field strength since this study is directed at merely demonstrating the effect of its presence. The response of this system to an applied electric field will be examined in each of the following experiments and details concerning all of the individual components employed are summarized in Table I.

The EFPN system shown in Fig. 1 was modified for some studies. In this modification, terminal $\mathrm{A}$ was replaced by a Plexiglas plate holding a brass ring which encircles the nebulizer and acts as the charging electrode. This modified device performed just as the original, but was judged safer to operate, because the area which is held at a high potential is greatly reduced.

\section{A. Light scattering measurements}

Light scattering observations were employed to evaluate the electric field's effect on the spatial distribution of the charged droplets as they are produced. Figure 2 portrays the experimental arrangement employed for these observations. A xenon arc lamp (cf. Table I) is used to illuminate the aerosols and a camera oriented just less than $180^{\circ}$ with respect to the light source records the scattered radiation as a function of applied electric field. The orientation of the photographic readout serves to maximize the Mie scattering signal and minimizes the effects of morphology (i.e., asphericity) of droplets.

To obtain a scattered light photograph, the air delivery pressure to the nebulizer is adjusted to a flow rate of approximately $2 \mathrm{l} / \mathrm{min}$; at this flow, a stable aspiration rate and aerosol cloud are produced. The xenon arc is then oriented so that its light strikes the aerosol just above the tip of the nebulizer. The camera is positioned to obtain maximum scattering signals but located so direct radiation from the lamp does not fall on it; photographs are then taken with and without the electric field being applied.

\section{B. Droplet size distribution measurements}

The most reliable method for determining the droplet size distribution produced by the EFPN system is the $\mathrm{MgO}$ impression technique. ${ }^{26}$ However, this technique yields no spatial information. The procedure involves coating microscope slides with a thin and uniform layer of fine-grain $\mathrm{MgO}$ and then collecting droplet impact impressions within the layer. Impressions are collected by quickly passing the coated slide through the aerosols formed both when the electric field is present and when it is absent. To measure each impression, photomicrographs were taken of groups of droplet impressions in a desired region of the slide; by comparison of these images with a photomicrograph of a calibrated reticle, taken with the same optical system, the size of each impression can be determined. Because each slide contains hundreds of droplet impressions, tabulation of the sizes measured from several slides provides a reliable and representative droplet size distribution. Droplets have been collected using this technique over a large size

TABLE I. Experimental components and conditions

\begin{tabular}{|c|c|}
\hline Beckman burner & $\begin{array}{l}\text { Model } 4030 \text { integral aspirator-burner (Beckman } \\
\text { Instruments, Inc., Fullerton, CA). }\end{array}$ \\
\hline $\begin{array}{l}\text { High voltage } \\
\text { source }\end{array}$ & $\begin{array}{l}\text { Fluke model } 408 \mathrm{~B} \text { high voltage dc power supply } \\
\text { operated at } 5.5 \mathrm{kV} \text { (John Fluke Mfg. Co., Inc., } \\
\text { Seattle, WA). }\end{array}$ \\
\hline $\begin{array}{l}\text { Light source used } \\
\text { in scattering } \\
\text { measurements }\end{array}$ & $\begin{array}{l}\text { 150-W xenon arc lamp (No. } 901 \mathrm{C}-11 \text {, Englehard- } \\
\text { Hanovia, Newark, NJ) with associated power } \\
\text { supply and housing (Bausch and Lomb, } \\
\text { Rochester, NY). Provided with fixed operat- } \\
\text { ing current. }\end{array}$ \\
\hline Current detection & $\begin{array}{l}\text { Keithley Instruments model } 414 \mathrm{~S} \text { picoammeter } \\
\text { (Keithley Instruments, Inc., Cleveland, } \mathrm{OH} \text { ). }\end{array}$ \\
\hline $\begin{array}{l}\text { Photographic } \\
\text { system }\end{array}$ & $\begin{array}{l}\text { Besseler Topcon Super D camera with a } f / 1.4 \\
\text { lens (Charles Besseler Company, E. Orange, } \\
\text { NJ) mounted onto a sturdy tripod. }\end{array}$ \\
\hline $\begin{array}{l}\text { Photomicrograph } \\
\text { system }\end{array}$ & $\begin{array}{l}\text { Topcon camera mounted onto a Unitron micro- } \\
\text { scope (model MKK-A4, Unitron Instrument } \\
\text { Co., Newton Highland, MA) via a Topcon } \\
\text { model III microscope attachment. Microscope } \\
\text { operated with } 10 \times \text { eyepiece and } 4 \times \text { objective. } \\
\text { Microscope slides illuminated by a Bausch and } \\
\text { Lomb microscope illuminator (Bausch and } \\
\text { Lomb Opt. Co., Rochester, NY). }\end{array}$ \\
\hline Gas handling & $\begin{array}{l}\text { Compressed air (Matheson Co.) supplied from } \\
\text { a single stage regulator (Air Products), con- } \\
\text { trolled by a needle valve (series } M \text {, Nupro } \\
\text { Co., Cleveland, OH) and monitored by a } \\
\text { Dwyer flowmeter (VFB, model No. 55- } \\
16027400 \text {, Dwyer Instruments, Inc., Michigan } \\
\text { City, IN). Flowrate while collecting droplet } \\
\text { impressions approximately } 1.21 / \mathrm{min} \text {, during } \\
\text { current measurement approximately } 2.1 \mathrm{l} / \mathrm{min} \\
\text { (corrected to STP). }\end{array}$ \\
\hline Solutions & $\begin{array}{l}\text { In all cases, the liquid being studied was } \\
\text { distilled water. }\end{array}$ \\
\hline
\end{tabular}


range and for a wide range of liquids and impact velocities ${ }^{26}$ and it has been shown that the technique is capable of measuring droplet diameters greater than $10 \mu \mathrm{m}$ with $3 \%-5 \%$ accuracy. ${ }^{2}$

\section{Droplet charge measurements}

Acquisition of charge is part of the mechanism controlling the reduction of a droplet's size, as described earlier. Also, the polarity and magnitude of a droplet's charge will influence that droplet's behavior upon interaction with other components in any spectrometer system. Therefore, an attempt was made to determine the charge that a droplet acquires from the EFPN device.

The average charge per droplet was deduced from the droplet size distribution, the nebulization rate, and the current that flows into the nebulized liquid stream. No measurable charge was induced onto the droplets in the absence of the electric field. Therefore, the current flowing into the nebulized stream, coupled with the nebulization rate (in $\mathrm{ml} / \mathrm{s}$ ) defines the charge that is acquired per unit volume of nebulized solution. In turn, the charged liquid is disrupted into a dispersion of droplets with fixed fractions existing in specific and statistically fixed size ranges which are defined by the droplet distribution data. The number of droplets in each size range formed per unit volume of liquid nebulized can then be calculated and the resulting charge per droplet determined.

Experimentally, the current was measured by connecting a picoammeter (cf. Table I) between terminal $\mathrm{B}$ and ground potential (terminal $B$ is disconnected from ground) and noting in which direction the current flows. Accurate measurement of the nebulization rate is accomplished with the aid of a simple apparatus which provides a solution reservoir kept at a constant level and a gas system which maintains a constant flow rate through the nebulizer. The amount of solution that is automatically added to keep a constant level in the reservoir during nebulization is accurately measured and corresponds to the quantity of solution that is nebulized. Recording the time period during which nebulization occurs results in the nebulization rate.

Apparatus used to maintain a constant level reservoir for the nebulizer consists of a single glass tube $15 \mathrm{~mm}$ i.d.), sealed on one end, and a partially filled $25-\mathrm{ml}$ graduated cylinder. In operation, the glass tube is filled with solution and quickly inverted, so its open end is just beneath the surface of the solution contained in the graduated cylinder; the cylinder then serves as the solution reservoir for the nebulizer. As liquid is drawn from the reservoir, the level of the solution in the graduated cylinder drops until the open end of the glass tube is exposed. At this point, an air bubble enters the glass tube and releases an identical volume of water into the reservoir. This process continues until the solution in the glass tube is exhausted. Therefore, this system maintains a relatively constant solution level in the reservoir, providing a constant nebulization rate, and the narrow

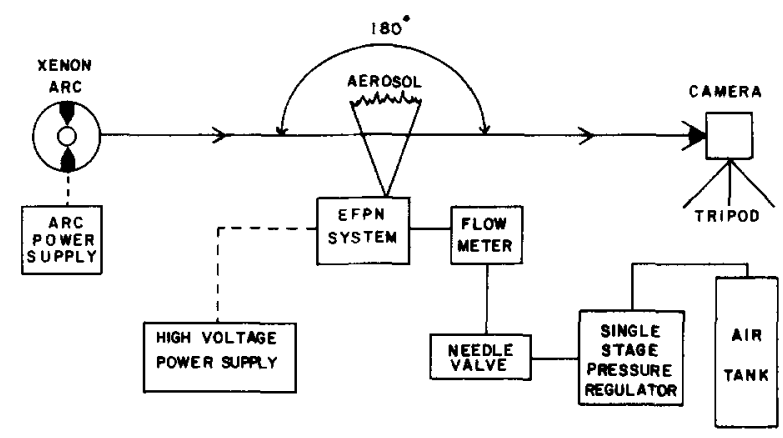

FIG. 2. Schematic diagram of light scattering observation apparatus $(\rightarrow-)$ optical path; (-- ) electrical path; $(\longrightarrow)$ gas flow.

gauge of the feed tube enables an accurate $( \pm 0 / .02 \mathrm{ml})$ determination of the volume nebulized.

\section{RESULTS AND DISCUSSION}

Photographs of scattered radiation qualitatively reveal the spatial distribution of the droplets produced by the EFPN system with and without the electric field. In turn, this distribution indicates both that the droplets are charged and that their size is reduced by the field's influence. These qualitative findings are confirmed through use of a direct and quantitative method for determining the droplet size distribution produced by the EFPN system. These results and their importance to practical flame and plasma spectrometry are discussed in this section.

\section{A. Effect of electric field on spatial distribution of droplets}

Light scattered from the aerosols produced by the EFPN system revealed a distinct effect of the electric field. The photograph in Fig. 3(a) depicts the scattered light profile when no electric field is present; the mainstream of the aerosol appears confined, with only a few droplets deviating from the axis of the aerosol. In contrast, Fig. 3(b) shows the change in nebulization which occurs when the field is applied. In Fig. 3(b), a much more diffuse aerosol cloud is found, with a large number of the droplets appearing to possess high velocities perpendicular to the primary axis of aerosol flow. It was qualitatively observed that the sedimentation rate of these outer droplets is extremely slow, suggesting that the droplets in the periphery of the aerosol, portrayed in Fig. 3(b), are very small. These results indicate that the same reduction in droplet size that has been characterized for charged liquid jets ${ }^{23}$ is probably occurring during electric-field pneumatic nebulization.

The acquisition of a charge by the droplets and the effect of the electric field on the charged droplets are vividly shown in the photograph in Fig. 3(b). The offaxis (peripheral) droplets in Fig. 3(b) are being strongly attracted to Terminal $A$ and collected there; hence they must be charged to the opposite polarity of the applied potential. Apparently, the dome-shaped region just above the tip of the nebulizer marks the interface be- 


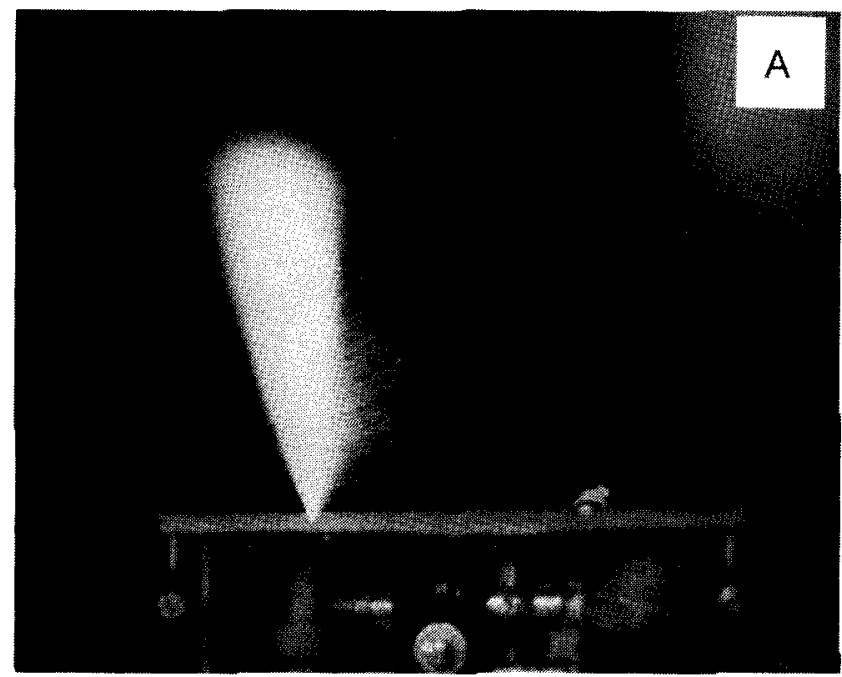

(a)

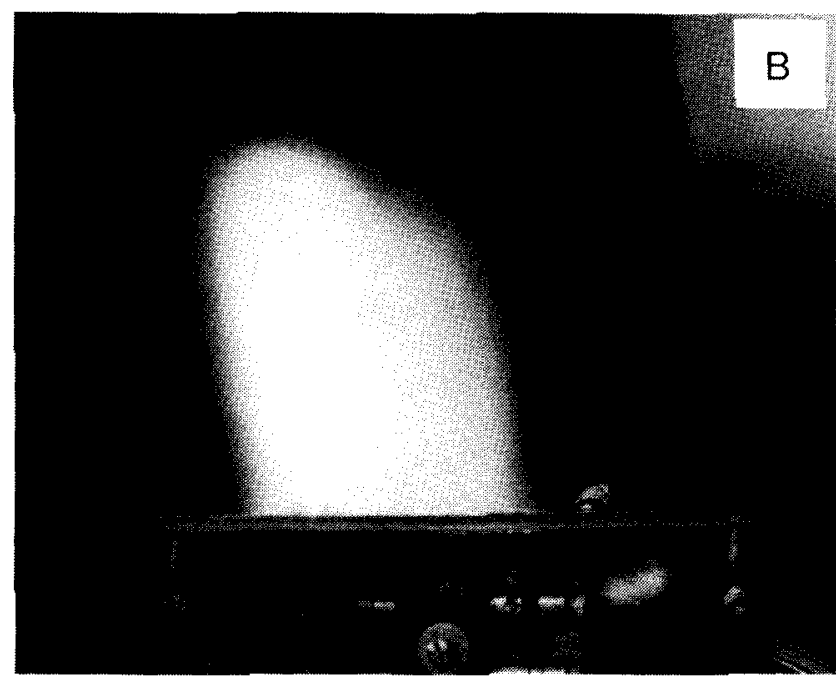

(b)

FIG. 3. Photographs of spatial light scattering profiles of aerosols produced by EFPN system. (a) - no electric field applied; (b) electric field applied.

tween the regions of opposite polarity. These effects are all observed to an identical degree when either positive or negative potentials are applied to the EFPN system.

The current flowing in the EFPN system upon application of the electric field is on the order of $4.4 \times 10^{-7} \mathrm{~A}$. Moreover, the direction of the current flow indicates that droplets are being charged to the polarity opposite from the applied potential, verifying the foregoing explanation given for the behavior of the droplets in Fig. 3(b). Significantly, the acquisition of such an excess surface charge can cause a droplet to become unstable, leading to its fragmentation. In effect, the charged droplet establishes a lower energy condition by decreasing its charge-to-surface area ratio through fragmentation into a number of small droplets. To cite a numerical example, a 40- $\mu$ m-diam droplet might disrupt into sixty-four $10-\mu \mathrm{m}$-diam droplets which then possess the same total volume. The average charge carried by
$40 \mu \mathrm{m}$ and $10 \mu \mathrm{m}$ droplets by the EFPN system can then be calculated from the measured current $(4.4$ $\times 10^{-7} \mathrm{~A}$ ), nebulization rate and droplet size distribution data, as previously described. When this calculation is performed, the surface charge on the $40 \mu \mathrm{m}$ and 10 $\mu \mathrm{m}$ droplets is found to be $1.7 \times 10^{-1.3}$ and $7.2 \times 10^{-16} \mathrm{C}$, respectively. In turn, these values indicate a charge-tosurface area ratio for the $10 \mu \mathrm{m}$ droplet which is an order of magnitude less than that for the $40 \mu \mathrm{m}$ droplet.

Unfortunately, highly charged droplets might also lead to a serious problem. Being highly charged, the droplets will possess a high affinity for any object at ground potential, including such areas as a nebulizer spray chamber and burner walls. Collection of a large quantity of droplets on these surfaces could lead to a substantial reduction in the amount of sample being introduced into the analytical source (i.e., flame or plasma). Of course, charging of the spray chamber and burner walls to a polarity the same as the droplet's could overcome this problem but might be impractical. Perhaps the best method for overcoming this potential problem would be to minimize the nebulizer and burner surface areas to which the droplets are exposed before they enter the flame or plasma.

\section{B. Effect of electric field on droplet size distribution}

Because the aerosols produced by the EFPN system are polydisperse (i.e., contain a range of droplet sizes), characterization of the aerosol requires knowledge of the droplet size distribution. In turn, to determine the droplet size distribution, one must accurately tabulate the frequency of occurrence of each droplet size. Numerous methods have been developed for measuring droplet sizes; the most general and convenient of these methods is the $\mathrm{MgO}$ impression method ${ }^{26}$ chosen for use in the present investigation.

More than 1500 droplet impressions were collected and measured from photomicrographs such as the one shown in Fig. 4. Measured droplet diameters were

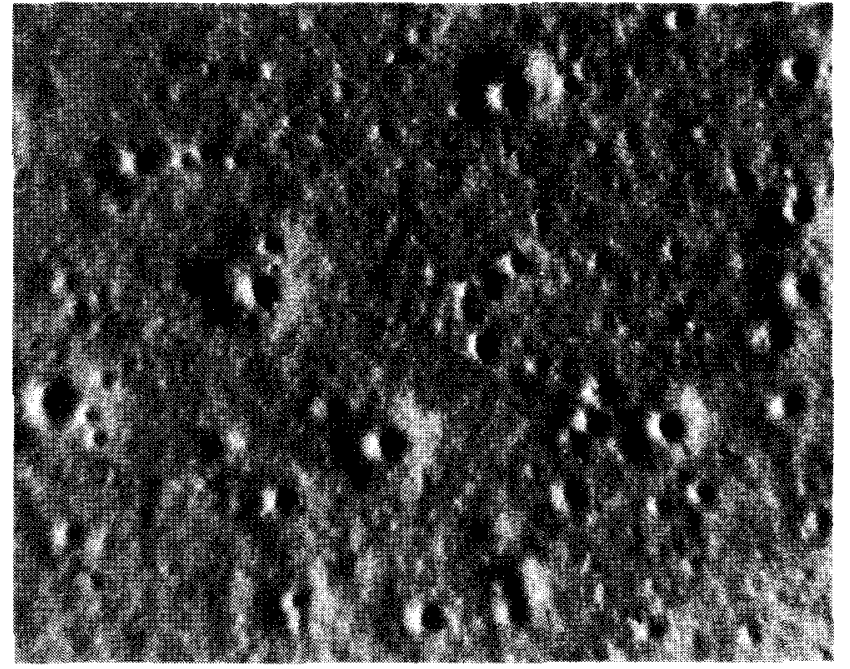

FIG. 4. Photomicrograph of droplet impressions in fine-grain magnesium oxide. 
placed into size groups which were centered around a nominal size and tabulated at $5-\mu \mathrm{m}$ intervals. All the droplet diameters which were measured to be less than $10 \mu \mathrm{m}$ were included in the $10-\mu \mathrm{m}$ size group. The efficiency of collection of droplets smaller than about $10 \mu \mathrm{m}$ is low, because such small droplets tend to follow the air flow around the $\mathrm{MgO}$-coated slides and are therefore not efficiently collected. Also, small droplets do not often leave measurable impressions upon impact, because of their low mass. Consequently, some error undoubtedly exists in the tabulated $10 \mu \mathrm{m}$ population; the same limitations prohibit the tabulation of any droplet sizes smaller than $5 \mu \mathrm{m}$. Overall, however, the $\mathrm{MgO}$ technique has been shown to be quite useful for characterizing aerosols of this nature. ${ }^{27}$ These tabulations are displayed as histograms in Fig. 5, where the percent of the total number of droplets observed is plotted against the diameter, in $\mu \mathrm{m}$, of each droplet size group. A distinct shift of the entire distribution towards smaller droplets occurs when the electric field is applied, proving that there is indeed a reduction in the size of the droplets produced by a pneumatic nebulizer under the influence of an electric field.

The mean droplet diameter of aerosols produced by nebulization of aqueous solutions with a Beckman nebulizer-burner has been shown to be approximately $20 \mu \mathrm{m} .{ }^{27}$ Consequently, it is worthwhile in the present study to examine the percent of the total number of droplets greater or less than $\mathbf{2 0} \mu \mathrm{m}$. Such a comparison, shown in Table II, demonstrates again the sharp decrease in droplet size that occurs when an electric field is applied. Significantly, the application of an electric field not only increases the number of small droplets but also provides a corresponding decrease in the number of large droplets. This finding agrees with the theoretical explanation given earlier concerning the mechanism of the electric field's effect upon pneumatic nebulization.

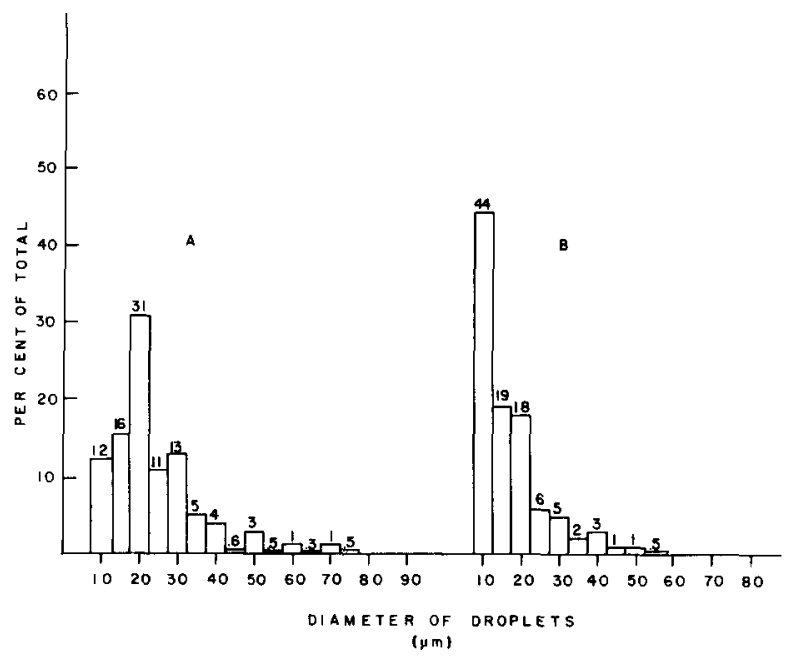

FIG. 5. Histogram representing droplet size distributions (see text for discussion). (a) - no electric field applied; (b) - electric field applied. The numbers centered above each bar represent the percent of the total number of droplets observed within that droplet size group.
TABLE II. Fraction of droplets which are greater or less than $20 \mu \mathrm{m}^{\mathrm{a}}$, produced by the EFPN system under no electric field and applied field conditions.

\begin{tabular}{ccc}
\hline \hline & $\begin{array}{c}\text { No electric } \\
\text { field applied }\end{array}$ & $\begin{array}{c}\text { Electric } \\
\text { field applied }\end{array}$ \\
\hline$\%<20 \mu \mathrm{m}$ & 28 & 63 \\
$\%>20 \mu \mathrm{m}$ & 41 & 19 \\
\hline
\end{tabular}

a The mean droplet diameter of aqueous aerosols produced by flameless Beckman burners has been determined to be $20 \mu \mathrm{m} .{ }^{27}$

$\mathrm{b}$ The nebulized solution in all cases was distilled water.

\section{Statistical evaluation of volume mean droplet diameter}

The representation of the droplet distribution data (cf. Fig. 5) by an empirical function enables one to quantitatively compare the measured distributions by computing their mean droplet diameters. Past studies ${ }^{28}$ have shown that the log-normal function is applicable to sprays of this nature. The log-normal function is statistically based and predicts that the natural logarithm of the droplet diameters will be distributed in a normal or Gaussian manner. Mugele and Evan ${ }^{28}$ found that an even better agreement with experiment could be realized by introducing an upper-limit parameter into the lognormal function. The upper-limit parameter simply represents the maximum stable droplet diameter; this realistic limit replaces the theoretical concept, implicit in the log-normal function, that there is an infinitely small probability that an infinitely large droplet might be formed. Examination of Fig. 5 demonstrates that an upper limit to the droplet diameter range can easily be defined.

The log-normal/upper-limit function was applied to the distribution data as described by Mugele and Evans. ${ }^{28}$ In this procedure, the logarithm of the droplet diameter is first plotted against the cumulative volume fraction on a probability scale; cumulative volume fractions are calculated for each droplet size group by first multiplying the frequency of occurrence by the volume of the droplet. The volume of the droplet is calculated assuming spherical geometry. By summing the volume of each previous droplet size group to the next group, one computes a cumulative volume for each group. Ratioing the cumulative volume computed for each group to the total cumulative volume results in a cumulative volume fraction for each group. In Fig. 6(a), droplet diameters are plotted on a logarithmic scale against cumulative volume fractions on a probability scale, for the case when the electric field is absent. The linearity of the plot, except at large diameters, demonstrates that the droplet distribution can be accurately represented by the log-normal function. The deviation from linearity at large droplet diameters apparent in Fig. 6(a) is inherent in the log-normal function and is corrected when the upper-limit parameter is included. Following the same procedure for the droplet data obtained when the electric field is present yields the plot shown in Fig. 6(b); as before, the plot demonstrates that the droplet size 


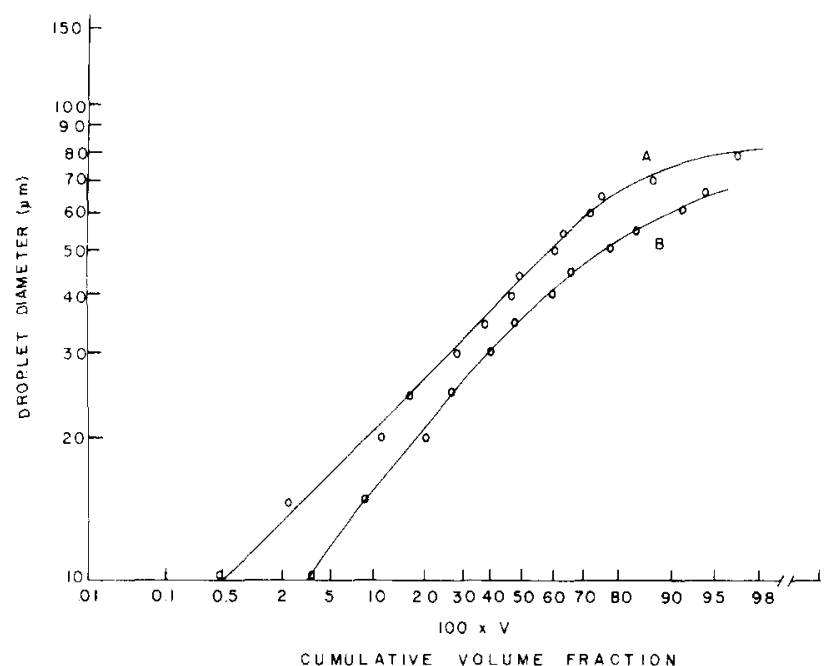

Fig. 6. Application of the log-normal function to the droplet distribution data. (a) no electric field applied; (b) electric field applied. Droplet diameters plotted on a logarithmic scale against cumulative volume fractions $(V)$ on a probability scale. Applied potential $=5.5 \mathrm{kV}$

distribution produced under the influence of the electric field can be represented by the log-normal function.

Mugele and Evans ${ }^{28}$ have derived an expression for the volume mean diameter $(\bar{X})$ of a droplet distribution which adheres to the log-normal and upper-limit functions:

$$
\bar{X}=X_{m}\left(1+2 a e^{1 / 4 \delta^{2}}+a^{2} e^{1 / \delta^{2}}\right)^{-1 / 2}
$$

In Eq. (2), the maximum stable droplet diameter, $X_{m}$, is the parameter associated with the upper-limit function and is determined from the plots in Figs. 6(a) and 6(b), as described by Carnes. ${ }^{29}$ The $a$ term in Eq. (2) is a dimensionless constant which is also readily determined from Figs. 6(a) and 6(b).$^{29}$ Finally, the size distribution parameter, $\delta$, which can be associated with how rapidly the distribution decreases from its maximum value, is obtained from the slopes of the lines in Figs. 6(a) and 6(b) (after the upper-limit function correction is applied). By calculating values for these parameters and solving Eq. (2), one can compute and compare volume mean diameters for the droplet distributions obtained both with and without the electric field being applied. Volume mean diameters are computed because droplets measured by impact techniques give volume averages.

The results of the foregoing mathematical treatment, summarized in Table III, reveal a pronounced decrease in the volume mean diameter upon application of the electric field, thereby substantiating the earlier predictions. The $95 \%$ confidence limits indicated in Table III reflect the accuracy of this statistical approach to droplet size measurement; a simple $t$-test confirmed that the listed mean values are significantly different.

The importance of the $63 \%$ decrease in the volume mean diameter of the droplets produced by the EFPN system (cf. Table III) is underscored by comparing it to the decrease in droplet size reported by Carnes ${ }^{27}$ for the nebulization of organic solvents. In these past
TABLE III. Results of the mathematical treatment of the droplet distribution data ${ }^{\mathrm{a}}$

\begin{tabular}{lcc}
\hline \hline & $\begin{array}{c}\text { No electric } \\
\text { field applied }\end{array}$ & $\begin{array}{c}\text { Electric } \\
\text { field applied }\end{array}$ \\
\hline $\begin{array}{l}\text { Maximum stable droplet } \\
\quad \text { diameter }\left(X_{m}\right)\end{array}$ & $125 \mu \mathrm{m}$ & $70 \mu \mathrm{m}$ \\
Dimensionless constant $(a)$ & 1.91 & 0.94 \\
Size distribution parameter $(\delta)$ & 0.92 & 0.52 \\
Volume mean diameter $(\bar{X})$ & $29.4 \pm 1.7 \mu \mathrm{m}^{\mathrm{b}}$ & $10.9 \pm 1.0 \mu \mathrm{m}$ \\
\hline
\end{tabular}

a Results for distilled water; see text for explanation of treatment.

b $\pm 95 \%$ confidence limits.

Applied voltage $=5.5 \mathrm{kV}$.

studies, ${ }^{27}$ only a $15 \%$ decrease in volume mean diameter was recorded when 4-methylpentan-2-one (MIBK) was nebulized instead of water. Therefore, one could expect a fourfold-greater reduction in droplet size when the EFPN system is employed instead of organic solvents; moreover, the convenience of using aqueous solutions is retained. Furthermore, even greater reductions in droplet size might arise if stronger electric fields were applied than those used here.

\section{ACKNOWLEDGMENTS}

This work was presented in part at the joint meeting of the Federation of Analytical Chemistry and Spectroscopy Societies (FACSS), XIX Colloquium Spectroscopicum Internationale, and 6th International Conference on Atomic Spectroscopy, Philadelphia, PA, Nov., 1976. Supported in part by the Office of Naval Research and by the National Science Foundation.

a) Author to whom correspondence should be sent.

${ }^{1}$ N. C. Clampitt and G. M. Hieftje, Anal. Chem. 44, 1211 (1972).

${ }^{2}$ G. J. Bastiaans and G. M. Hieftje, Anal. Chem. 46, 901 (1974).

3 Y. Tanasawa, Trans. Soc. Mech. Eng. Jpn. 6, S10 (1940).

${ }^{4}$ R. L. Peskin and R. J. Raco, J. Acoust. Soc. Am. 35, 1378 (1963).

H. Straubel, Mikrochim. Acta 1955, 329.

${ }^{6}$ R. Mavrodineanu and H. Boiteux, Flame Spectroscopy (Wiley Interscience, New York, 1965), pp. 85-112.

${ }^{7}$ R. Herrmann and C. Th. J. Alkemade, Chemical Analysis by Flame Photometry (Wiley-Interscience, New York. 1963), pp. 20-27.

\& S. Nukiyama and Y. Tanasawa, Trans. Soc. Mech. Eng. Jpn. 5, S15 (1939); Chem. Abstr. 35, 4258 (1941).

"Lord Rayleigh, Proc. Lond. Math. Soc., 10, 4 (1878).

${ }^{10} \mathrm{~S}$. Nukiyama and Y. Tanasawa, Trans. Soc. Mech. Eng. Jpn. 4, S13 (1938).

1 E. G. Richardson, Appl. Sci. Res. A 4, 374 (1954)

12 V. B. Fainerman, J. Appl. Chem. (USSR) 49, 565 (1976).

${ }^{13}$ G. M. Schmid, R. S. Hurd, and E. S. Snavely, Jr., J. Electrochem. Soc. 109, 852 (1962)

14 A. B. Basset, Am. J. Math. 16, 13 (1894).

15 G. Taylor, Proc. R. Soc. London A280, 383 (1964).

16 J. M. Schneider, N. R. Lindblad, C. D. Hendricks, and J. M. Crowley, J. Appl. Phys. 38, 2599 (1967).

17 J. D. Shorley and D. Michelson, Nucl. Instrum. Methods 82, 295 (1970).

18 A. L. Huebner and H. N. Chu, J. Fluid Mech. 49, 361 (1971).

${ }^{19}$ G. Osamu, J. Electrochem. Soc. Jpn. 24, 229 (1956).

${ }^{20}$ R. H. Magarvey and L. E. Outhouse, J. Fluid Mech. 13, 151 (1962).

21 J. J. Hogan, R. S. Carsons, J. M. Schneider, and C. D. Hendricks, AIAA J 2, 1460 (1965).

${ }^{22}$ F. J. Weinberg, Proc. R. Soc. London A307, 195 (1968).

23 A. L. Huebner, J. Fluid Mech. 38, 679 (1969).

${ }^{24}$ A. L. Huebner, Science 168, 118 (1970)

25 E. Barreto, Aerosol Sci. 2, 219 (1971)

26 K. R. May, J. Sci. Instrum. 22, 187 (1945)

27 J. A. Dean and W. J. Carnes, Anal. Chem. 34, 192 (1962).

${ }^{28}$ R. A. Mugele and H. D. Evans, Ind. Eng. Chem. 43, 1317 (1951).

29 W. J. Carnes, Ph.D. thesis (University of Tennessee, 1961). 